\title{
A Scalable Two-Phase Top-Down Specialization Approach for Data Anonymization using MapReduce on Cloud
}

\author{
Ranjana Nadagoudar ${ }^{1}$, Radhika ${ }^{2}$ \\ Associate Professor, Dept. of Computer Science and Engineering, VTU RO Centre, Kalaburgi, India ${ }^{1}$ \\ IV Sem M. Tech, Dept. of Computer Science and Engineering, VTU RO Centre, Kalaburgi, India ${ }^{2}$
}

\begin{abstract}
A large number of cloud forces require users to carve up private data like electronic health records for data analysis or mining, bringing privacy concerns. Anonymizing data sets via generalization to satisfy certain privacy requirements such as k-anonymity is a widely used category of privacy preserving techniques. At present, the scale of data in many cloud applications increases massively in accordance with the Big Data trend, thereby making it a challenge for commonly used software tools to confine, manage, and process such large-scale data within a adequate elapsed time. As a result, it is a challenge for existing anonymization approaches to accomplish privacy preservation on privacy-sensitive large-scale data sets due to their insufficiency of scalability. In this paper, we propose a scalable two phase top-down specialization (TDS) to anonymize large-scale data sets using the MapReduce framework on cloud. In both phases of our approach, we deliberately design a group of inventive MapReduce jobs to concretely accomplish the specialization computation in a highly scalable way. Experimental assessment results demonstrate that with our approach, the scalability and efficiency of TDS can be significantly enhanced over existing approaches.
\end{abstract}

Keywords: Data anonymization, top-down specialization, MapReduce, cloud, privacy preservation

\section{INTRODUCTION}

CLOUD computing, a disruptive trend at present, certain increasing information is exposed to data users for poses a considerable impact on current IT industry and various analysis and mining. A variety of anonymization research communities [1]. Cloud computing provides algorithms with different anonymization operations have massive computation power and storage capacity via been proposed [15]. However, the scale of data sets that utilizing a large number of commodity computers together, need anonymizing in some cloud applications increases enabling users to organize applications cost-effectively tremendously in accordance with the cloud computing and without heavy infrastructure asset. Cloud users can reduce Big Data trends [1]. Data sets have become so large that huge upfront investment of IT infrastructure, and focus on anonymizing such data sets is becoming a considerable their own core business. However, numerous possible challenge for conventional anonymization algorithms. The customers are still diffident to take advantage of cloud due researchers have begin to investigate the scalability to privacy and security concerns [5]. The research on cloud problem of large-scale data anonymization. Large-scale privacy and security has come to the depiction [9]. Privacy data processing frameworks like MapReduce have been is one of the most concerned issues in cloud computing, included with cloud to provide dominant computation and the concern aggravates in the perspective of cloud capability for applications. So, it is promising to adopt computing although some privacy issues are not new[1], such frameworks to address the scalability problem of [5]. Personal data like electronic health records and anonymizing large-scale data for privacy preservation. In financial transaction records are usually deemed extremely our research, we leverage MapReduce, a widely adopted sensitive although these data can present significant human analogous data processing framework, to address the benefits if they are analyzed and mined by organizations scalability problem of the top-down specialization (TDS) such as disease research centres. Data privacy can be approach [12] for large-scale data anonymization. The divulged with less effort by malevolent cloud users or TDS approach, offering a good trade off between data providers because of the failures of some conventional effectiveness and data reliability, is widely applied for data privacy protection measures on cloud [5]. This can bring anonymization [12]. Most TDS algorithms are centralized, substantial economic loss or strict social reputation resulting in their insufficiency in handling large scale data mutilation to data owners. Hence, data privacy issues need sets. Although some dispersed algorithms have been to be addressed urgently before data sets are analyzed or proposed they mainly focus on secure have been proposed pooled on cloud. Data anonymization has been extensively they mainly focus on secure anonymization of data sets studied and widely adopted for data privacy preservation from numerous parties, rather than the scalability aspect. in non interactive data publishing and sharing scenarios As the MapReduce computation hypothesis is moderately [11]. Data anonymization refers to hiding characteristics simple, it is still a challenge to design proper MapReduce and/or sensitive data for owners of data records. Then, the jobs for TDS. In this paper, we propose a highly scalable privacy of an individual can be effectively preserved while two-phase TDS approach for data anonymization based on 
MapReduce on cloud. To make full use of the parallel information from one party to another. Jurczyk and Xiong capability of MapReduce on cloud, specializations [25] and Mohammed et al. [20] proposed distributed required in an anonymization process are split into two algorithms to anonymize horizontally partitioned data sets phases. In the first one, original data sets are partitioned retained by multiple holders. However, the above into a group of smaller data sets, and these data sets are distributed algorithms mainly aim at securely integrating anonymized in parallel, producing intermediate results. In and anonymizing multiple data sources. Our research the second one, the intermediate results are integrated into mainly focuses on the scalability issue of TDS one, and further anonymized to achieve consistent k- anonymization, and is, therefore, orthogonal and anonymous data sets. We leverage MapReduce to complementary to them. As to MapReduce-relevant accomplish the concrete computation in both phases. A privacy protection, Roy et al. [26] investigated the data group of MapReduce jobs is purposely designed and privacy problem caused by MapReduce and presented a corresponding to perform specializations on data sets system named Airavat incorporating mandatory access collaboratively.

control with differential privacy. Further, Zhang et al. [27]

The major contributions of our research are threefold. leveraged MapReduce to automatically partition a First, we cratively apply MapReduce on cloud to TDS for computing job in terms of data security levels, protecting data anonymization and consciously design a group of data privacy in hybrid cloud. Our research exploits innovative MapReduce jobs to concretely accomplish MapRedue itself to anonymize large-scale data sets before thespecializations in a highly scalable fashion. Second, we data are further processed by other MapReduce jobs, propose a two-phase TDS approach to gain high scalability arriving at privacy preservation.

via allowing specializations to be conducted on several data partitions in parallel during the first phase. Third, experimental results show that our approach can significantly improve the scalability and efficiency of TDS for data anonymization over existing approaches. The remainder of this paper is organized as follows: The next section reviews related work, and analyzes the scalability problem in existing TDS algorithms.

\section{B.Problem Analysis}

We analyze the scalability problem of existing TDS approaches when handling large-scale data sets on cloud. The centralized TDS approaches in [12], [20], and [21] exploits the data structure TIPS to improve the scalability and efficiency by indexing anonymous data records and retaining statistical information in TIPS. The data structure The remainder of this paper is organized as follows: The speeds up the specialization process because indexing next section reviews related work, and analyzes the structure avoids frequently scanning entire data sets and scalability problem in existing TDS algorithms. In Section storing statistical results circumvents recomputation III, we briefly present proposed system for our approach. overheads. On the other hand, the amount of metadata Section IV preliminary approach, and Section 5 formulates retained to maintain the statistical information and linkage the two-phase TDS approach. In Section 6 formulates information of record partitions is relatively large mapreduce version of centralized TDS. Finally, we compared with data sets themselves, thereby consuming conclude this paper.

\section{II. . RELATED WORK AND PROBLEM ANALYSIS}

\section{A. Related Work}

Recently, data privacy preservation has been extensively investigated [11]. We briefly review related work below. LeFevre et al. [17] addressed the scalability problem of anonymization algorithms via introducing scalable decision trees and sampling techniques. Iwuchukwu and Naughton [18] proposed an R-tree indexbased approach by building a spatial index over data sets, achieving high efficiency. However, the above approaches aim at multidimensional generalization [15], thereby failing to work in the TDS approach. Fung et al. [12], [20], [21] proposed the TDS approach that produces anonymous data sets without the data exploration problem [11]. A data structure Taxonomy Indexed PartitionS (TIPS) is subjugated to improve the efficiency of TDS. But the approach is centralized, leading to its insufficiency in handling large-scale data sets. Several distributed algorithms are proposed to preserve privacy of multiple data sets retained by multiple parties. Jiang and Clifton [24] and Mohammed et al. [22] proposed distributed algorithms to anonymize vertically partitioned data from different data sources without disclosing privacy considerable memory. Moreover, the overheads incurred by maintaining the linkage structure and updating the statistic information will be huge when date sets become large. Hence, centralized approaches probably suffer from low efficiency and scalability when handling large-scale data sets. There is an assumption that all data processed should fit in memory for the centralized approaches [12]. Unfortunately, this assumption often fails to hold in most data-intensive cloud applications nowadays. In cloud environments, computation is provisioned in the form of virtual machines (VMs). Usually, cloud compute services offer several flavors of VMs. As a result, the centralized approaches are difficult in handling large-scale data sets well on cloud using just one single VM even if the VM has the highest computation and storage capability. A distributed TDS approach [20] is proposed to address the distributed anonymization problem which mainly concerns privacy protection against other parties, rather than scalability issues. Further, the approach only employs information gain, rather than its combination with privacy loss, as the search metric when determining the best specializations. As pointed out in [12], a TDS algorithm without considering privacy loss probably chooses a specialization that leads to a quick violation of anonymity requirements. Hence, the distributed algorithm fails to produce anonymous data sets exposing the same data 
utility as centralized ones. Besides, the issues like expected, resulting in privacy breach. The k-anonymity communication protocols and fault tolerance must be kept privacy model can combat such a privacy breach because in mind when designing such distributed algorithms. As it ensures that an individual will not be distinguished from such, it is inappropriate to leverage existing distributed other at least $\mathrm{k}-1$ ones. The anonymity parameter $\mathrm{k}$ is algorithms to solve the scalability problem of TDS.

\section{III.PROPOSED SYSTEM} specified by users according to their privacy requirements. In the TDS approach, a data set is anonymized via performing specialization operations. A specialization

In this paper, we propose a scalable two-phase top-down operation is to replace a domain value with all its child specialization (TDS) approach to anonymize large-scale values. Formally, a specialization operation is represented data sets using the MapReduce framework on cloud. In as spec $: p \rightarrow$ Child(p), where $p$ is a domain value and both phases of our approach, we deliberately design a Child(p) DOM is the set of all the child values of p. The group of innovative MapReduce jobs to concretely domain values of an attribute form a "cut" through its accomplish the specialization computation in a highly taxonomy tree [11]. The cut of attribute Attri, denoted as scalable way. This approach get input data's and split into Cuti, $1 \leq \mathrm{i} \leq \mathrm{m}$, is a subset of values in DOMi . Cuti the small data sets. Then we apply the contains exactly one value in each root-to-leaf path in ANONYMIZATION on small data sets to get intermediate taxonomy tree TTi. The cuts of all attributes determine the result. Then small data sets are merge and again apply the anonymity of a data set. To capture the degree of ANONYMIZATION. We analyze the each and every data anonymization intuitively during the specialization set sensitive field and give priority for this sensitive field. process, we give the subsequent definition.

Then we apply ANONYMIZATION on this sensitive field only depending upon the scheduling.

\section{A.ADVANTAGES OF PROPOSED SYSTEM}

- Accomplish the specializations in a highly scalable fashion.

- Gain high scalability.

- $\quad$ Significantly improve the scalability and efficiency of TDS for data anonymization over existing approaches.

- The overall performance of the providing privacy is high.

- Its ability to handles the large amount of dat sets.

- The anonymization is effective to provide the privacy on data sets.

- $\quad$ Here we using the scheduling strategies to handle the high amount of datasets.

\section{A.Basic Notations}

\section{IV.PRELIMINARY}

We describe several basic notations for convenience. Let D anonymity is violated, to expose the maximum data utility. denote a data set containing data records. A record $r € D$ The goodness of a specialization is measured by a search has the form $r=(v 1, v 2, \ldots, v m, s v)$, where $\mathrm{m}$ is the metric. We adopt the information gain per privacy loss number of attributes, vi, $1 \leq i \leq m$, is an attribute value and (IGPL), a trade off metric that considers both the privacy $s v$ is a sensitive value like diagnosis. The set of sensitive and information requirements, as the search metric in our values is denoted as SV. An attribute of a record is denoted approach. A specialization with the highest IGPL value is as Attr, and the taxonomy tree of this attribute is denoted as regarded as the best one and selected in each round.

TT. Let DOM represent the set of all domain values in TT. Given a specialization spec $: p \rightarrow$ Child(p), the IGPL of The quasi-identifier of a record is denoted as qid $=$ hq1, the specialization is calculated by q2, . . . qmi, where qi $€$ DOMi. Quasi identifiers, IGPL $(\mathrm{spec})=\operatorname{IG}(\mathrm{spec})=(\operatorname{PL}(\mathrm{spec})+1)$. representing groups of anonymous records, can lead to The term IG(spec) is the information gain after performing privacy breach if they are too specific that only a small spec, and PL(spec) is the privacy loss. IG(spec) and group of people are linked to them [11]. Quasi-identifier PL(spec) can be computed via statistical information set is denoted as QID $=($ Attr1, Attr2, . . Attrm $)$. The set derived from data sets.

of the records with qid is defined as QI-group [28], denoted by QIG(qid). QI is the acronym of quasiidentifier.Without loss of generality, we adopt k-anonymity $[23]$ as the privacy model herein, i.e., for any qid $€$ QID, the size of G(qid) must be zero or at least k. Otherwise, the individuals owning such a quasi-identifier can be linked to sensitive information with higher confidence than

\section{TWO-PHASE TOP DOWN SPECIALIZATION(TPTDS)}

A.Sketch of Two-Phase Top-Down Specialization

We propose a TPTDS approach to conduct the computation required in TDS in a highly scalable and efficient fashion. The two phases of our approach are 
based on the two levels of parallelization provisioned by 4: Execute MRTDS(D, k, ALI) $\rightarrow$ AL* to achieve kMapReduce on cloud. Basically, MapReduce on cloud has anonymity.

two levels of parallelization, i.e., job level and task level. 5: Specialize D according to AL*, Output D*. Job level parallelization means that multiple MapReduce jobs can be executed simultaneously to make full use of In essential, TPTDS divides specialization operations cloud infrastructure resources. Combined with cloud, required for anonymization into the two phases. Let SP1i, MapReduce becomes more powerful and elastic as cloud $1 \leq \mathrm{i} \leq \mathrm{p}$, denote the specialization sequence on Di in the can offer infrastructure resources on demand, for example, first phase, i.e., SP1i = (speci1, speci2, . . . ; speciji ), Amazon Elastic MapReduce service [29]. Task level where ji is the number of specializations. The first parallelization refers to that multiple mapper/reducer tasks common subsequence of SP1i, $1 \leq \mathrm{i} \leq \mathrm{p}$, is denoted as SPI in a MapReduce job are executed simultaneously over data . Let SP2 denote the specialization sequence in the second splits. To achieve high scalability, we parallelizing phase. SP2 is determined by ALI rather than kI . multiple jobs on data partitions in the first phase, but the Specifically, more specific ALI implies smaller SP2. resultant anonymization levels are not identical. To obtain Throughout TPTDS, the specializations in the set SPI $\mathrm{v}$ finally consistent anonymous data sets, the second phase is SP2 come into effect for anonymization. The influence of necessary to integrate the intermediate results and further $\mathrm{p}$ and $\mathrm{kI}$ on the efficiency is analyzed as follows. Greater $\mathrm{p}$ anonymize entire data sets. Details are formulated as means higher degree of parallelization in the first phase, follows. In the first phase, an original data set $\mathrm{D}$ is and less $\mathrm{kI}$ indicates more computation is conducted in the partitioned into smaller ones.Then, we run a subroutine first phase. Thus, greater $\mathrm{p}$ and less kI can improve the over each of the partitioned data sets in parallel to make efficiency.

full use of the job level parallelization of MapReduce. The subroutine is a MapReduce version of centralized TDS B.Data Partition

(MRTDS) which concretely conducts the computation When $\mathrm{D}$ is partitioned into Di, $1 \leq \mathrm{i} \leq \mathrm{p}$, it is required in TPTDS. MRTDS anonymizes data partitions to required that the distribution of data records in Di is generate intermediate anonymization levels. An similar to D.A data record here can be treated as a point in intermediate anonymization level means that further an m-dimension space, where $m$ is the number of specialization can be performed without violating $\mathrm{k}$ attributes. Thus, the intermediate anonymization levels anonymity. MRTDS only leverages the task level derived from Di, $1 \leq \mathrm{i} \leq \mathrm{p}$, can be more similar so that we parallelization of MapReduce. Formally, let function can get a better merged anonymization level. Random $\operatorname{MRTDS}(\mathrm{D}, \mathrm{k}, \mathrm{AL}) \rightarrow \mathrm{AL0}$ represent a MRTDS routine sampling technique is adopted to partition D, which can that anonymizes data set D to satisfy k-anonymity from satisfy the above requirement. Specifically, a random anonymization level AL to AL0. Thus, a series of functions number rand, $1 \leq$ rand $\leq p$, is generated for each data $\operatorname{MRTDS}(\mathrm{Di}, \mathrm{kI}, \mathrm{AL0}) \rightarrow \mathrm{AL0i}, 1 \leq \mathrm{i} \leq \mathrm{p}$, are executed record. A record is assigned to the partition Drand. simultaneously in the first phase. The term kI denotes the Algorithm 2 shows the MapReduce program of data intermediate anonymity parameter, usually given by partition. Note that the number of Reducers should be application domain experts. Note that kI should satisfy kI equal to p, so that each Reducer handles one value of rand, $\geq \mathrm{k}$ to ensure privacy preservation. AL0 is the initial exactly producing p resultant files. Each file contains a anonymization level, i.e., AL0 $=(\{$ Top 1$\},\{$ Top2 $\}, \ldots$, random sample of D.

$\{$ Topm $\})$, where Topj $€ \mathrm{DOMj}, 1 \leq \mathrm{j} \leq \mathrm{m}$, is the topmost domain value in TTi. ALO $\mathrm{i}$ is the resultant intermediate ALGORITHM 2. DATA PARTITION MAP \& anonymization level. In the second phase, all intermediate REDUCE.

anonymization levels are merged into one. The merged Input: Data record (IDr, r), $\mathrm{r} € \mathrm{D}$, partition parameter $\mathrm{p}$. anonymization level is denoted as ALI . The merging Output: Di, $1 \leq \mathrm{i} \leq \mathrm{p}$.

process is formally represented as function Map: Generate a random number rand, where $1 \leq \mathrm{rand} \leq \mathrm{p}$; merge $((\mathrm{AL01}, \mathrm{AL02}, \ldots$;AL0p) $) \rightarrow$ ALI .Then, the whole emit (rand, r).

data set D is further anonymized based on ALI, achieving Reduce: For each rand, emit (null, list(r)). Once partitioned $\mathrm{k}$-anonymity finally, i.e., MRTDS(D, $\mathrm{k}, \mathrm{ALI}) \rightarrow \mathrm{AL}^{*}$, where data sets $\mathrm{Di}, 1 \leq \mathrm{i} \leq \mathrm{p}$, are obtained, we run MRTDS(Di, $\mathrm{AL}^{*}$ denotes the final anonymization level.

\section{ALGORITHM 1. SKETCH OF TWO-PHASE TDS (TPTDS).}

$\mathrm{kI}$, AL0) on these data sets in parallel to derive intermediate anonymization levels $A L i, 1 \leq i \leq p$.

\section{Anonymization Level Merging}

All intermediate anonymization levels are merged into one Input: Data set D, anonymity parameters $\mathrm{k}, \mathrm{kI}$ and the in the second phase. The merging of anonymization levels number of partitions $\mathrm{p}$.

Output: Anonymous data set $D^{*}$.

1: Partition D into Di, $1 \leq \mathrm{i} \leq \mathrm{p}$.

2: Execute MRTDS(Di, kI, AL0) $\rightarrow$ AL0i, $1 \leq \mathrm{i} \leq \mathrm{p}$ in parallel as multiple MapReduce jobs.

3: Merge all intermediate anonymization levels into one,

Merge(AL01, AL02, . ., AL0p) $\rightarrow$ ALI .

is completed by merging cuts. Specifically, let Cuta in ALOa and Cutb in ALOb be two cuts of an attribute. There exist domain values qa $€$ Cuta and $q b €$ Cutb that satisfy one of the three conditions: qa is identical to qb, qa is more general than $q b$, or qa is more specific than qb. To ensure that the merged intermediate anonymization level ALI never violates privacy requirements, the more general one 
is selected as the merged one, for example, qa will be Initialization, except that it requires less computation and selected if qa is more general than or identical to qb. For consumes less network bandwidth.

the case of multiple anonymization levels, we can merge Since the IGPL Update job dominates the scalability and them in the same way iteratively. The following lemma efficiency of MRTDS, we briefly analyze its complexity as ensures that ALI still complies privacy requirements.

\section{D.Data Specialization}

follows. Let $\mathrm{n}$ denote all the records in a data set, $\mathrm{m}$ be the number of attributes, $s$ be the number of mappers, and $t$ be the number of reducers. As a mapper emits $(m+1)$ key-

An original data set $D$ is concretely specialized for value pairs, it takes $O(1)$ space and $O(m+n / s)$ anonymization in a one-pass MapReduce job. After time.Similarly, a reducer takes $\mathrm{O}(1)$ space and $\mathrm{O}(\mathrm{m}+\mathrm{n} / \mathrm{t})$ obtaining the merged intermediate anonymization level time. Note that a reducer only needs $\mathrm{O}(1)$ space due to the ALI, we run MRTDS(D, k, ALI) on the entire data set D, MapReduce feature that the key-value pairs are sorted in and get the final anonymization level AL* Then, the data the shuffle phase. Otherwise, the reducer needs more space set $\mathrm{D}$ is anonymized by replacing original attribute values to accumulate statistic information for a variety of in $\mathrm{D}$ with the responding domain values in $\mathrm{AL}^{*}$. Details of specializations. The communication $\operatorname{cost}$ is $\mathrm{O}(\mathrm{m}+\mathrm{n})$ Map and Reduce functions of the data specialization according to the map function, but communication traffics MapReduce job are described in Algorithm3. The Map can be reduced heavily by optimization techniques like function emits anonymous records and its count. The Combiner.

Reduce function simply aggregates these anonymous records and counts their number. An anonymous record and its count represent a QI-group. The QI-groups constitute the final anonymous data sets.

\section{ALGORITHM 3. DATA SPECIALIZATION MAP \& REDUCE.}

Input: Data record (IDr, r), $\mathrm{r} €$ D. ; Anonymization level $\mathrm{AL}^{*}$.

Output: Anonymous record ( $\mathrm{r}^{*}$, count).

Map: Construct anonymous record $\mathrm{r}^{*}=\mathrm{p} 1,(\mathrm{p} 2, \ldots, \mathrm{pm}$, sv), pi, $1 \leq \mathrm{i} \leq \mathrm{m}$, is the parent of a specialization in current $\mathrm{AL}$ and is also an ancestor of vi in r, emit (r,count). Reduce: For each ${ }^{*}$, sum $\leftarrow \sum$ count, emit ( ${ }^{*}$, sum).

\section{MAPREDUCE VERSION OF CENTRALIZED TDS}

We elaborate the MRTDS in this section. MRTDS plays a core role in the two-phase TDS approach, as it is invoked in both phases to concretely conduct computation. Basically, a practical MapReduce program consists of Map and Reduce functions, and a Driver that coordinates the macro execution of jobs.

\section{A. MRTDS Driver}

Usually, a single MapReduce job is inadequate to accomplish a complex task in many applications. Thus, a group of MapReduce jobs are orchestrated in a driver program to achieve such an objective. MRTDS consists of MRTDS Driver and two types of jobs, i.e., IGPL Initialization and IGPL Update. The driver arranges the execution of jobs.

\section{B. IGPL Initialization Job}

The main task of IGPL Initialization is to initialize information gain and privacy loss of all specializations in the initial anonymization level AL.

\section{C.IGPL Update Job}

The IGPL Update job dominates the scalability and efficiency of MRTDS, since it is executed iteratively. So far, iterative MapReduce jobs have not been well supported by standard MapReduce framework like Hadoop. The IGPL Update job is quite similar to IGPL

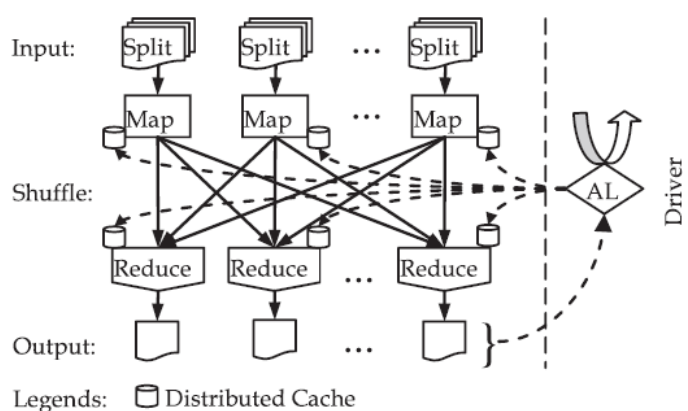

Fig.1. Execution framework overview of MRTDS

\section{D.Implementation and Optimization}

To elaborate how data sets are processed in MRTDS, the execution framework based on standard MapReduce is depicted in Fig. 1. The solid arrow lines represent the data flows in the canonical MapReduce framework. From Fig. 1, we can see that the iteration of MapReduce jobs is controlled by anonymization level AL in Driver. The data flows for handling iterations are denoted by dashed arrow lines. AL is dispatched from Driver to all workers including Mappers and Reducers via the distributed cache mechanism. The value of $\mathrm{AL}$ is modified in Driver according to the output of the IGPL Initialization or IGPL Update jobs. As the amount of such data is extremely small compared with data sets that will be anonymized, they can be efficiently transmitted between Driver and workers. We adopt Hadoop, an open-source implementation of MapReduce, to implement MRTDS. Since most of Map and Reduce functions need to access current anonymization level AL, we use the distributed cache mechanism to pass the content of $\mathrm{AL}$ to each Mapper or Reducer node as shown in Fig. 1. Also, Hadoop provides the mechanism to set simple global variables for Mappers and Reducers. The best specialization is passed into the Map function of IGPL Update job in this way. The partition hash function in the shuffle phase is modified because the two jobs require that the key-value pairs with the same key:p field rather than entire key should go to the same Reducer. To reduce communication traffics, MRTDS exploits combiner mechanism that aggregates the keyvalue pairs with the same key into one on the nodes running Map functions. 


\section{CONCLUSION}

In this paper, we have investigated the scalability problem of large-scale data anonymization by TDS, and proposed a highly scalable two-phase TDS approach using MapReduce on cloud. Data sets are partitioned and anonymized in parallel in the first phase, producing intermediate results. Then, the intermediate results are merged and further anonymized to produce consistent $\mathrm{k}$ anonymous data sets in the second phase.

We have creatively applied MapReduce on cloud to data anonymization and intentionally designed a group of inventive MapReduce jobs to concretely achieve the specialization computation in a highly scalable way. The scalability and efficiency of TDS are improved significantly over existing approaches.

\section{ACKNOWLEDGMENT}

I would like to express my sence of proud gratitude and indebtedness to my guide, for his valuable guidance, suggestions, timely supervision for successful completion of my paper. Above all I would like to thanks all my family members and friends for their constructive critism and construct support in making this grand success.
[15] K. LeFevre, D.J. DeWitt, and R. Ramakrishnan, "MondrianMultidimensional K-Anonymity," Proc. 22nd Int'l Conf. Data Eng. (ICDE '06), 2006

[16] V. Borkar, M.J. Carey, and C. Li, "Inside 'Big Data Management': Ogres, Onions, or Parfaits?," Proc. 15th Int'l Conf. Extending Database Technology (EDBT '12), pp. 3-14, 2012

[17] K. LeFevre, D.J. DeWitt, and R. Ramakrishnan, "Workload-Aware Anonymization Techniques for Large-Scale Data Sets," ACM Trans. Database Systems, vol. 33, no. 3, pp. 1-47, 2008.

[18] T. Iwuchukwu and J.F. Naughton, "K-Anonymization as Spatial Indexing: Toward Scalable and Incremental Anonymization," Proc. 33rd Int'l Conf. Very Large Data Bases (VLDB '07), pp.746- 757,2007.

19] J. Dean and S. Ghemawat, "Mapreduce: Simplified Data rocessing on Large Clusters," Comm. ACM, vol. 51, no. 1, pp. 107-113, 2008.

[20] N. Mohammed, B. Fung, P.C.K. Hung, and C.K. Lee, "Centralized and Distributed Anonymization for High-Dimensional Healthcare Data," ACM Trans. Knowledge Discovery from Data, vol. 4, no.4 Article 18, 2010

[21] B. Fung, K. Wang, L. Wang, and P.C.K. Hung, "PrivacyPreserving Data Publishing for Cluster Analysis," Data and Knowledge Eng., vol. 68, no. 6, pp. 552-575, 2009.

[22] N. Mohammed, B.C. Fung, and M. Debbabi, "Anonymity Meets Game Theory: Secure Data Integration with MaliciousParticipants," VLDB J., vol. 20, no. 4, pp. 567-588, 2011.

[23] L. Sweeney, "k-Anonymity: A Model for Protecting Privacy," Int'l J. Uncertainty, Fuzziness and Knowledge-Based Systems,vol. 10, no. 5 , pp. 557-570, 2002.

24] W. Jiang and C. Clifton, "A Secure Distributed Framework for Achieving k-Anonymity,” VLDB J., vol. 15, no. 4, pp. 316-333, 2006

\section{REFERENCE}

[1] S. Chaudhuri, "What Next?: A Half-Dozen Data Management Research Goals for Big Data and the Cloud," Proc. 31st Symp. Principles of Database Systems (PODS '12), pp. 1-4, 2012

[2] M. Armbrust, A. Fox, R. Griffith,, Joseph, R. Katz, A Konwinski, G. Lee, D. Patterson, A. Rabkin, I. Stoica, and M. Zaharia, "A View of Cloud Computing," Comm. ACM, vol. 53, no. 4 , pp. $50-58,2010$

[3] L. Wang, J. Zhan, W. Shi, and Y. Liang, "In Cloud, Can Scientific Communities Benefit from the Economies of Scale?, IEEE Trans. Parallel and Distributed Systems, vol. 23, no. 2, pp.296-303, Feb. 2012

[4] H. Takabi, J.B.D. Joshi, and G. Ahn, "Security and Privacy Challenges in Cloud Computing Environments," IEEE Security and Privacy, vol. 8, no. 6, pp. 24-31, Nov. 2010

[5] D. Zissis and D. Lekkas, "Addressing Cloud Computing Security Issues," Future Generation Computer Systems, vol. 28, no. 3, pp. 583- 592, 2011

[6] X. Zhang, C. Liu, S. Nepal, S. Pandey, and J. Chen, "A Privacy Leakage Upper-Bound Constraint Based Approach for CostEffective Privacy Preserving of Intermediate Data Sets in Cloud," IEEE Trans. Parallel and Distributed Systems, to be published, 2012.

[7] L. Hsiao-Ying and W.G. Tzeng, "A Secure Erasure Code-Based Cloud Storage System with Secure Data Forwarding," IEEE Trans. Parallel and Distributed Systems, vol. 23, no. 6, pp. 995- 1003, 2012.

[8] N. Cao, C. Wang, M. Li, K. Ren, and W. Lou, "Privacy-Preserving Multi-Keyword Ranked Search over Encrypted Cloud Data," Proc. IEEE INFOCOM, pp. 829-837, 2011.

[9] P. Mohan, A. Thakurta, E. Shi, D. Song, and D. Culler, "Gupt: Privacy Preserving Data Analysis Made Easy," Proc. ACM SIGMOD Int'l Conf. Management of Data (SIGMOD '12), pp. 349- 360, 2012.

[10] Microsoft HealthVault, http://www.microsoft.com/health/ww/ products/Pages/healthvault.aspx, 2013.

[11] B.C.M. Fung, K. Wang, R. Chen, and P.S. Yu, "Privacy-Preserving Data Publishing: A Survey of Recent Devel- opments," ACM Computing Surveys, vol. 42, no. 4, pp. 1-53, 2010.

[12] B.C.M. Fung, K. Wang, and P.S. Yu, "Anonymizing Classification Data for Privacy Preservation," IEEE Trans. Knowledge and Data Eng., vol. 19, no. 5, pp. 711-725, May 2007.

[13] X. Xiao and Y. Tao, "Anatomy: Simple and Effective Privacy Preservation," Proc. 32nd Int'l Conf. Very Large Data Bases (VLDB'06), pp. 139-150, 2006

[14] K. LeFevre, D.J. DeWitt, and R. Ramakrishnan, "Incognito: Efficient Full-Domain K-Anonymity," Proc. ACM SIGMOD Int'l Conf. Management of Data (SIGMOD ’05), pp. 49-60, 2005. 